\title{
Optomechanic interaction in a corrugated phoxonic nanobeam cavity
}

\author{
Mourad Oudich, ${ }^{1, *}$ Said El-Jallal, ${ }^{1,2}$ Yan Pennec, ${ }^{1}$ Bahram Djafari-Rouhani, ${ }^{1}$ Jordi Gomis-Bresco, ${ }^{3}$ Daniel Navarro-Urrios,${ }^{3, \dagger}$ \\ Clivia M. Sotomayor Torres, ${ }^{3}$ Alejandro Martínez, ${ }^{4}$ and Abdelkader Makhoute ${ }^{2}$ \\ ${ }^{1}$ Institut d'Electronique, de Microélectronique et de Nanotechnologie, UMR CNRS 8520, Université Lille 1, Villeneuve d'Ascq, France \\ ${ }^{2}$ Physique du Rayonnement et de l'Interaction Laser Matière, Université de Moulay Ismail, Meknès, Morocco \\ ${ }^{3}$ Catalan Institute of Nanotechnology, Campus UAB, 08193 Bellaterra, Barcelona, Spain \\ ${ }^{4}$ Nanophotonics Technology Center, Universidad Politécnica de Valencia,Valencia, Spain \\ (Received 18 February 2014; revised manuscript received 6 May 2014; published 18 June 2014)
}

\begin{abstract}
The interaction between phonons and photons is investigated theoretically in a phoxonic cavity inside a corrugated nanobeam waveguide presenting band gaps for both electromagnetic and elastic waves. The structure is made by drilling periodic holes on a silicon nanobeam with lateral periodic stubs and the tapered cavity is constructed by changing gradually the geometrical parameters of both the holes and stubs. We show that this kind of cavity displays localized phonons and photons inside the gaps, which can enhance their interaction and also promotes the presence of many optical confined modes with high quality factor. Using the finite-element method, we demonstrate that with appropriate design of the tapering construction, one can control the cavity modes frequency without altering significantly the quality factor of the photonic modes. By changing the tapering rates over the lattice constants, we establish the possibility of shifting the phononic cavity modes frequency to place them inside the desired gap, which enhances their confinement and increases the mechanical quality factor while keeping the strength of the optomechanic coupling high. In our calculations, we take account of both mechanisms that contribute to the acousto-optic interaction, namely photoelastic and interface motion effects. We show that in our case, these two effects can contribute additively to give high coupling strength between phononic and photonic cavity modes. The calculations of the coupling coefficient which gives the phonon-photon coupling strength give values as high as $2 \mathrm{MHz}$ while photonic cavity modes display quality factor of $10^{5}$ and even values up to $3.4 \mathrm{MHz}$ but with smaller photonic quality factors.
\end{abstract}

DOI: 10.1103/PhysRevB.89.245122

PACS number(s): 43.40.+s, 42.70.Qs, 42.79.Jq

\section{INTRODUCTION}

The design of highly dispersive media to control the propagation of both electromagnetic and acoustic waves has received increasing interest during the last few years. This particularly concerns periodic structures called photonic $[1,2]$ and phononic [3-5] crystals which have respectively the capability to control and manipulate light and sound waves. These periodic structures can exhibit absolute band gaps that make them very efficient tools for wave energy trapping and guiding, opening the possibility for many potential applications in wave guiding, filtering, confinement, and sensing. Recently, there have been a growing interest in the so-called Phoxonic crystals (PxC) which exhibit simultaneously photonic and phononic band gaps [6-13], thus allowing the confinement of both excitations in a cavity or a waveguide which is the necessary condition to enhance their interaction for the purpose of acousto-optic (AO) interaction [13-17]. Phoxonic crystals can be then promising as optomechanical structures where $\mathrm{AO}$ coupling can be enhanced to provide new ways for controlling light with acoustic waves.

AO interaction has been widely studied in different optomechanical structures. High quality cavities in slabs have been designed and proven to be efficient to provide strong phononphoton coupling. Safavi-Naeini et al. [18] observed a strong

\footnotetext{
*Present address: Institut Jean Lamour, Université de Lorraine, Vandoeuvre-lès-Nancy, F-54500, France; mourad.oudich@ univ-lorraine.fr

${ }^{\dagger}$ Present address: NEST, Istituto Nanoscienze - CNR and Scuola Normale Superiore, Piazza San Silvestro 12, Pisa, I-56127, Italy.
}

AO coupling between co-localized resonant phonons and photons inside a well-tailored tapered linear cavity in a silicon slab. Using membrane acoustic wave, Fuhrman et al. [19] were able to demonstrate experimentally the acoustic modulation of the optical wavelength of a confined mode inside a cavity. Other works have reported theoretically and experimentally on strong AO coupling inside the optomechanical slab cavity [20]. AO coupling has also been studied in nanobeams where strong interaction between phonon and photons has been evidenced both in theory and experiments. Eichenfield et al. [21,22] fabricated an optomechanical silicon nanobeam with periodic design shape in which localized phonons and photons created inside a cavity defect provide strong AO coupling. Recently, Chan et al. [23] studied the phonon-photon interaction in a tapered strip nanobeam with holes. Most of these works involve photonic cavity modes without necessarily trapping localized phononic modes in a cavity using complete band gap. We have recently demonstrated that high phonon-photon coupling can be achieved by localizing the modes inside the gaps [17] using a cavity in a two-dimensional (2D) infinite $\mathrm{PxC}$ and that the modes' symmetry is important and must be taken into account when looking for the suitable coupling of a pair of phonon and photon [24,25]. In fact, we have found out that whatever the symmetry of the photonic mode, the coupling occurs only if the acoustic mode displays even symmetry wave field distribution with respect to the cavity geometrical shape $[24,25]$. We pointed out this condition both in 2D infinite and slab phoxonic cavities with different shapes.

Another important feature in the optomechanical device is the quality factor (QF) for both phononic and photonic cavity modes involved in the AO interaction. In the experimental 
(a)

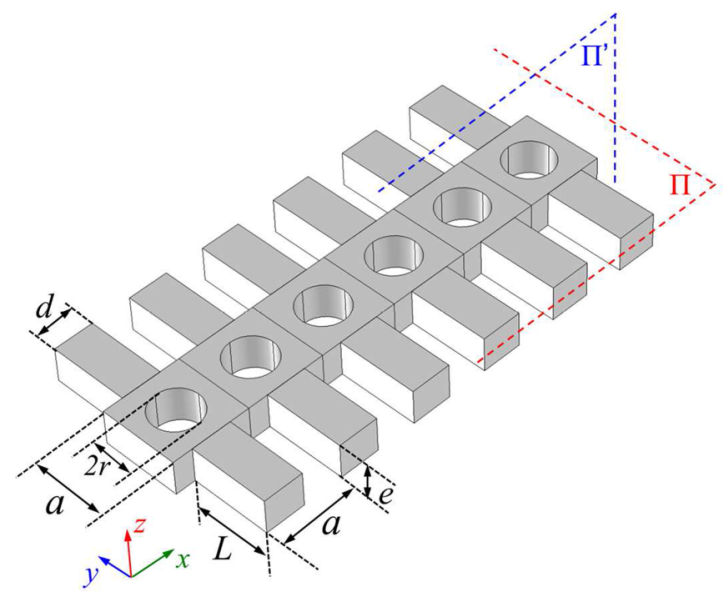

(b)

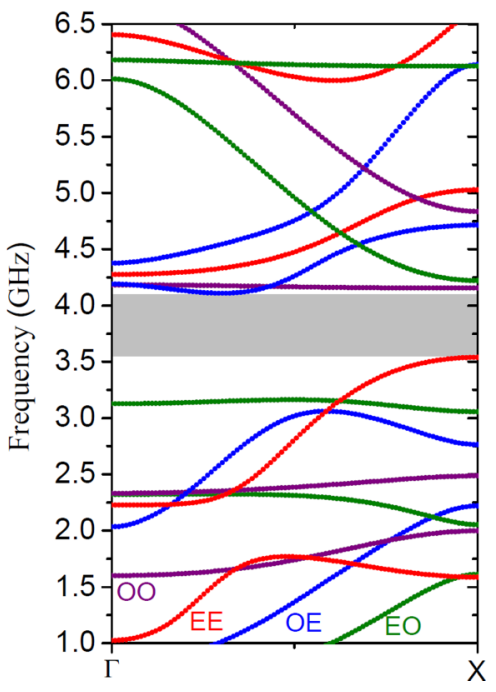

FIG. 1. (Color online) (a) Periodic corrugated silicon nanobeam with parallelepiped stubs grafted on each side and circular holes drilled in the middle. The radius of the holes, the thickness of the nanobeam, the stubs' length and width are respectively denoted $r, e, L$, and $d$ and fixed to $r=0.3 a, e=0.44 a, L=a$, and $d=0.5 a$, where $a$ is the lattice parameter fixed to $500 \mathrm{~nm}$. (b) Nanobeam's phononic band structure for wave propagation in $x$ direction, in which we highlighted the modes with the symmetry EO, EE, OO, and OE in green, red, purple, and blue colors respectively. The gray region indicates the absolute band gap.

point of view, a high QF cavity photon is needed to achieve and observe optomechanical coupling [23,26]. The design of cavities providing such a property is a challenging task and has been the object of many conceptual and technological works [27-32]. In all the previous works on optomechanical structures, smart designs of photonic cavities have been proposed for high QF photonic cavity modes as it is the basement of efficient experimental coupling characterization.

In this work we study theoretically the AO interaction in cavity defects created inside a corrugated phoxonic nanobeam with periodic holes [Fig. 1(a)]. Indeed, we have shown that the holes and the corrugations are respectively favorable to create the photonic and phononic band gaps [33], so this structure is suitable to provide simultaneous phononic and photonic gaps. Furthermore, both confined acoustic and optical modes can be found even within a simple cavity obtained by creating a silicon space between two holes [33]. However, the quality factor of the confined optical modes in such a simple cavity remained poor (less than $10^{3}$ ). So, we proposed a tapered cavity in which the period and hole sizes are changed progressively over several cells from the perfect crystal towards the cavity center. The fabrication of such samples allowed finding confined modes with very high acousto-optic coupling rates and quality factors (QFs) [34]. An interesting feature of the present paper is to investigate more elaborate geometries and design of the tapered cavity in the above nanobeam such that the phononic modes fall inside appropriate band gaps. We make a careful analysis of the acoustic modes' symmetry to identify those giving the best optomechanical coupling inside the gaps. More particularly, we shall discuss how the frequencies of the localized phonons can be tuned by changing the size of the stubs in the tapered cavities to make them fall into a partial band gap with the appropriate symmetry or even into a complete band gap. In Ref. [21] (see supplementary information therein) Eichenfield et al. shows that modes coupling between localized and leaky phonon modes can significantly limit the mechanical quality factor. The aim of the proposed structures here is to design cavities that ensure the confinement of the phononic modes while preventing their leakage out of the cavity and, in parallel, keeping or improving the strength of the optomechanical coupling.

Two mechanisms contribute to the optomechanic interaction, namely the photoelastic effect and the deformation of the interfaces due to the acoustic strain. Our calculations take account of both mechanisms and estimate the strength of the AO coupling in two different but complementary ways. The first one is the calculation of the so-called coupling coefficient based on the overlap between the acoustic and optical fields [23-25]. In the second method, we calculate the modulation of the photonic cavity mode due to the acoustic mode. Namely, the wavelength of the photonic mode in the deformed cavity is calculated at several instants of the acoustic period as we have described in recent previous works $[17,18,28]$. Based on our recent works [24,25], we have shown that only phononic modes which are of even symmetry with respect to both symmetry planes of the nanobeam (one horizontal and one vertical plane) are suitable to provide nonvanishing optomechanical interaction, thus allowing the search of confined modes inside gaps of particular even symmetry rather than only in the absolute band gaps.

The paper is organized as follows: in the first section we remind of the phoxonic strip waveguide structure as well as the band gaps that can provide for different phononic and photonic modes with specific symmetry of the nanobeam. Section II presents our tapering design of the cavities with the investigation of the existing confined phonons and photons modes inside the band gaps. Section III makes the investigation of the AO coupling involving both photoelastic PE and moving 
interface (MI) effects by using two approaches to estimate their strength contributions in the whole optomechanical coupling. AO coupling results are presented and discussed for different kinds of tapered nanobeam cavities. We finish this paper by highlighting the main conclusions over the obtained results.

\section{PHOXONIC STRIP WAVEGUIDE}

The phoxonic structure studied in this paper is made up of a straight silicon nanobeam waveguide combined with a periodic repetition of symmetric parallelepiped stubs grafted on each side and circular holes drilled in the middle [see Fig. 1(a)]. It was demonstrated that this strip waveguide provides simultaneously an absolute phononic band gap and a partial photonic gap for modes having the electromagnetic fields with symmetry odd and even respectively with respect to the symmetry planes $\Pi^{\prime}(x z$ plane) and $\Pi$ ( $x y$ plane) [Fig. 1(a)] [33]. The photonic modes with this specific symmetry will be called OE modes (odd-even). In this whole study, the lattice parameter is fixed to $a=500 \mathrm{~nm}$ and the other geometrical parameters were chosen [see Fig. 1(a)] to make the OE photonic wavelength gap range from 1486 to $1855 \mathrm{~nm}$. We remind also that the photonic modes we are dealing with are transverse magnetic (TM) like waves as their electric field is mainly in the $x-y$ plane and their magnetic field is along the perpendicular direction $z$.

In the phononic band structure presented in Fig. 1(b), we have highlighted with different colors the elastic modes having specific symmetry along the two symmetry planes $\Pi$ ' and $\Pi$ of the strip waveguide. The mode having even (E) symmetry with respect to $\Pi^{\prime}$ and odd $(\mathrm{O})$ symmetry to $\Pi$ are called EO modes and are indicated in green in the band structure. We distinguish also the modes EE (even to $\Pi^{\prime}$ and even to $\Pi$ ), OO (odd to $\Pi^{\prime}$ and odd to $\Pi$ ), and OE (odd to $\Pi^{\prime}$ and even to $\Pi$ ) presented in red, purple, and blue colors respectively in Fig. 1(b). We can then notice the absolute band gap which ranges from 3.54 to $4.11 \mathrm{GHz}$ indicated by the gray region in the figure. We can also distinguish partial gaps associated to each symmetry $\mathrm{OO}$, $\mathrm{EE}, \mathrm{OE}$, or EO of the modes. The reason behind this symmetry distinction between the modes is important when searching for acoustic modes that can couple with the photonic modes OE studied here.

To make this phoxonic structure more useful as an optomechanical tool, we have conducted a study over the design of a new phoxonic cavity capable of confining both electromagnetic and acoustic waves inside the gaps to enhance their interaction together with a high QF.

Regarding the finite-element method, we used Comsol Multiphysics software to calculate the band structures and optical and acoustic wave fields of the trapped cavity modes. From these results, a script allows the calculation of coupling coefficients using the expressions reported by Chan et al. [23]. Concerning the OM coupling evaluation from the modulation of the photonic cavity mode's wavelength during one acoustic period, we used also the finite-element method to calculate the monochromatic steady-state photonic mode by applying a given acoustic strain field. The acoustic period is sampled into several time steps, and the photonic cavity modes are calculated in the perturbed structure at each time step. This procedure enables us to obtain the evolution of the photonic mode wavelength during one acoustic period. The finite-element method was also used to perform optical and mechanical transmission calculation for the QF evaluation.

\section{TAPERED PHOXONIC CAVITY}

To achieve high QF phoxonic cavity, a tapered cavity was constructed where the size of the unit cell is varying progressively from the center of the cavity towards both sides. More precisely, the unit cell at the center of the cavity is smaller than the normal unit cell in the crystal and then the sizes of the neighboring unit cells progressively increase from the cavity center towards each side to reach the normal unit cell [Fig. 2(a)]. It was demonstrated that this kind of constructive cavity can be very efficient to increase the QF of the trapped light $[32,35]$. In this section, we present the design of such a tapered cavity when the center of the cavity is in between two holes [Fig. 2(a)] and the lattice parameter decreases with a parabolic shape as shown in Fig. 2(b). In this example, two different parabolic taperings, called $\alpha$ and $\beta$ [Fig. 2(b)], are presented. For all these tapered phoxonic cavities, the radius of the holes as well as the width of the lateral stubs are decreased from cell to cell successively as the periodicity decreases according to the relation $r=0.3 a$ and $d=0.5 a$, where $r$ is the holes' radius and $d$ the stubs' width [Fig. 1(a)].

First, we investigate inside the photonic gap the OE modes that are confined within the cavity and display a high QF. For the two above cavities, at least six photonic cavity modes were found with OE symmetry and having TM-like polarization. In the following, we shall only focus on one of them that gives the most relevant results in terms of $\mathrm{AO}$ interaction strength. Figure 3 presents the distribution of the electric and magnetic fields of this photonic mode as well as the transmission coefficient through the nanobeam with tapering $\alpha$ and $\beta$.

As we can see from Fig. 3(a), the considered TM-like photonic cavity mode is well confined inside the tapered region of the structure. It has an even symmetry along the

(a)

Tapered cavity
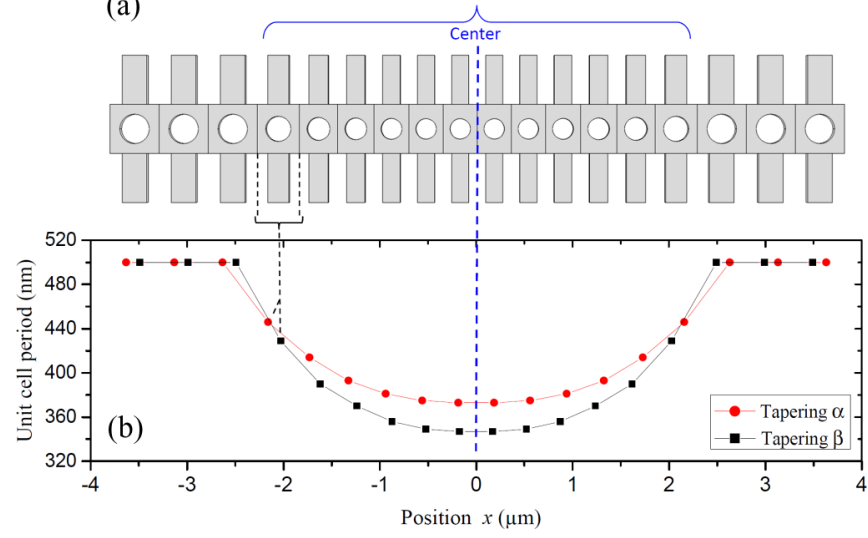

FIG. 2. (Color online) (a) Schematic view of the tapered cavity created inside the phoxonic strip waveguide where the center is between two holes. (b) Unit-cell period profile from cell to cell outside and inside the cavity as a function of the spatial position. Two parabolic shapes called tapering $\alpha$ and $\beta$ are plotted respectively with red circles and black squares. 
(a)

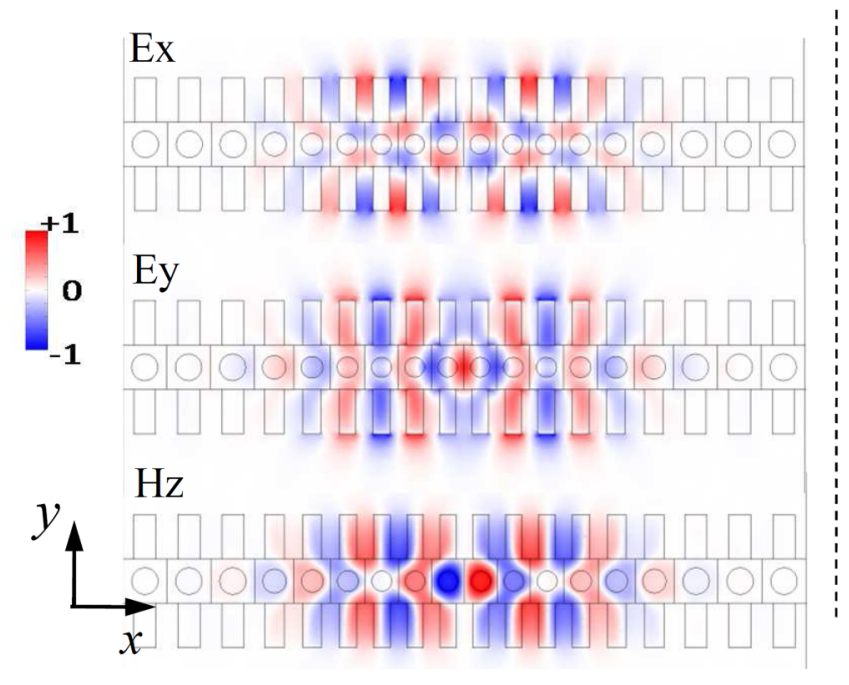

(b)
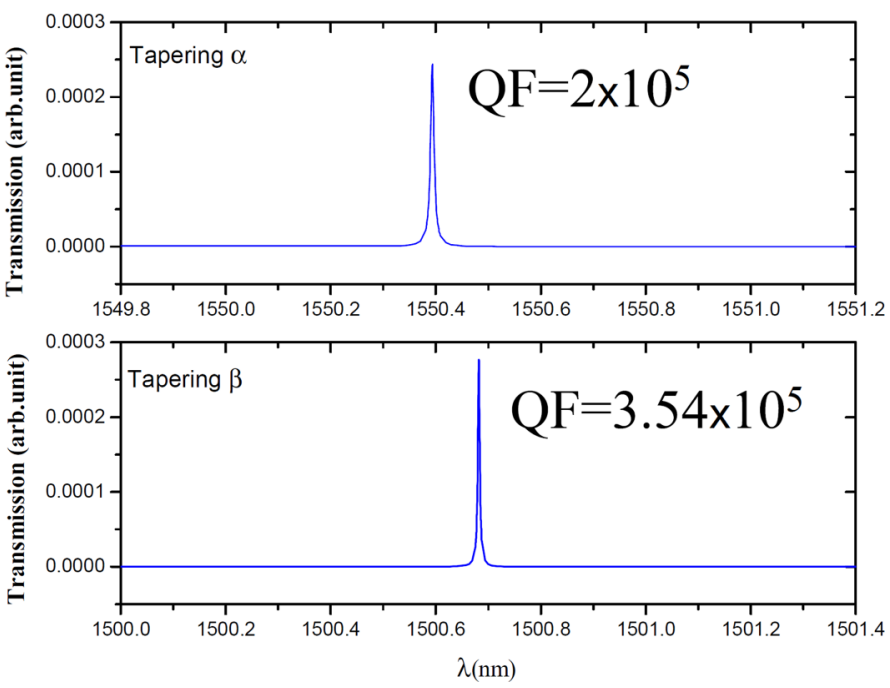

FIG. 3. (Color online) (a) Distribution of the electric and magnetic fields of the OE cavity photonic mode selected for the calculation of the AO interaction. (b) Transmission coefficient through the structure showing the peak of the cavity mode in the two cases of tapering $\alpha$ and $\beta$.

$y-z$ plane cutting the middle of the cavity in between two holes. Transmission calculations allow an estimation of the $\mathrm{QF}$ of the photonic mode for the two considered taperings $\alpha$ and $\beta$. In the case of tapering $\alpha$, the photonic mode is located at exactly $1550.4 \mathrm{~nm}$ with a QF of $2 \times 10^{5}$ whereas the same mode is found at $1500.68 \mathrm{~nm}$ in the case of tapering $\beta$ with higher QF of $3.54 \times 10^{5}$. These values of QF are less than $10^{7}$ reported in previous works $[18,21,23]$ but remain highly sufficient for experimental characterization [34]. One can then notice that the change in tapering gives the possibility of shifting the wavelength of the optical mode while the QF remains high. This can be explained by the change of the effective optical index inside the cavity which is higher in the center of the cavity in the case of tapering $\beta$ as compared to tapering $\alpha$.

To study the AO interaction in the case of this photonic cavity mode, we calculate the phononic dispersion and focused on localized acoustic modes inside the tapered defect that gives the strongest AO coupling.

\section{ESTIMATION OF THE OPTOMECHANIC COUPLING}

The AO coupling is quantified theoretically by calculating the photonic wavelength shift caused by the structure mechanical motion introduced by the phonon. This shift is estimated by taking into account both mechanisms that contribute to the coupling, namely the photoelastic (PE) effect and the moving interfaces (MI) effect as we pointed out before [17,23-25,36]. We remind that the PE effect is a result of the acoustic strain inside the silicon bulk which changes the dielectric permittivity while the MI mechanism comes from the dielectric permittivity variation in the vicinity of the silicon-vacuum boundaries caused by their acoustical motion. Also, the strength of the phonon-photon coupling is estimated by two equivalent approaches for both PE and MI effects: the time modulation of the photon frequency by the phonon where the AO coupling strength given by the amplitude of photonic wavelength modulation by the acoustical motion, and the coupling coefficients calculation which quantifies the photonic frequency shift induced by the zero-point motion of the mechanical field of the phonon for each PE and MI effect [17,24,25,36]. For the coupling coefficients, the OM quantification is done using the formulations given by Chan et al. [23] for the two effects:

$$
\begin{gathered}
g_{\mathrm{PE}}=-\frac{\omega}{2} \frac{\langle E|\delta \varepsilon| E\rangle}{\int_{\mathrm{V}} \mathbf{E} \cdot \mathbf{D} d V} \sqrt{\hbar / 2 M_{\mathrm{eff}} \Omega}, \\
g_{\mathrm{MI}}=-\frac{\omega}{2} \frac{\oint_{\partial \mathrm{V}}(\mathbf{U} \cdot \mathbf{n})\left(\Delta \varepsilon\left|\mathbf{E}_{\|}\right|^{2}-\Delta \varepsilon^{-1}\left|\mathbf{D}_{\perp}\right|^{2}\right) d S}{\int_{\mathrm{V}} \mathbf{E} \cdot \mathbf{D} d V} \sqrt{\hbar / 2 M_{\mathrm{eff}} \Omega,}
\end{gathered}
$$

where $\mathbf{U}$ is the normalized displacement field $(\max \|\mathbf{U}\|=1)$, $\mathbf{n}$ is the outside normal to the boundary, $\mathbf{E}$ is the electric field, and $\mathbf{D}$ is the electric displacement field. $\varepsilon$ is the dielectric permittivity, $\Delta \varepsilon=\varepsilon_{\text {silicon }}-\varepsilon_{\text {air }}$ and $\Delta \varepsilon^{-1}=\varepsilon_{\text {silicon }}^{-1}-\varepsilon_{\text {air }}^{-1}$. $\delta \varepsilon_{i j}=-\varepsilon_{0} n^{4} p_{i j k l} S_{k l}$, where $p_{i j k l}$ are the photoelastic tensor components, $n$ is the refractive index of silicon, and $S_{k l}$ the strain tensor components. $\omega$ and $\Omega$ are the optical and acoustic frequencies respectively and $M_{\text {eff }}$ is the effective motional mass related to the normalized acoustic displacement field by $M_{\text {eff }}=\rho \int_{\mathrm{V}}\|\mathbf{U}\|^{2} d V$, where $\rho$ is the silicon density.

The total optomechanical coupling coefficient $g$ is then given by

$$
g=g_{\mathrm{PE}}+g_{\mathrm{MI}} .
$$

Furthermore, using the symmetry of the structure, we have shown that only acoustic modes having even symmetry wave field can couple to the photonic modes. So in our case, the phononic modes with EE symmetries with respect to the planes $\Pi$ and $\Pi^{\prime}$ [Fig. 1(a)] are to couple with the optical modes, in particular in the case of OE photonic modes considered in the previous section.

Besides the symmetries with respect to the planes $\Pi$ and $\Pi^{\prime}$, the cavity displays also another symmetry plane, namely 
(a)

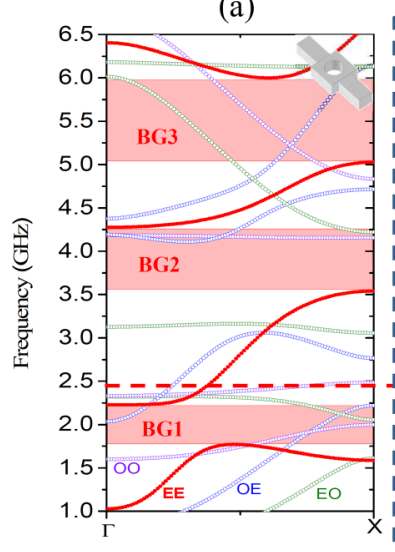

(b)

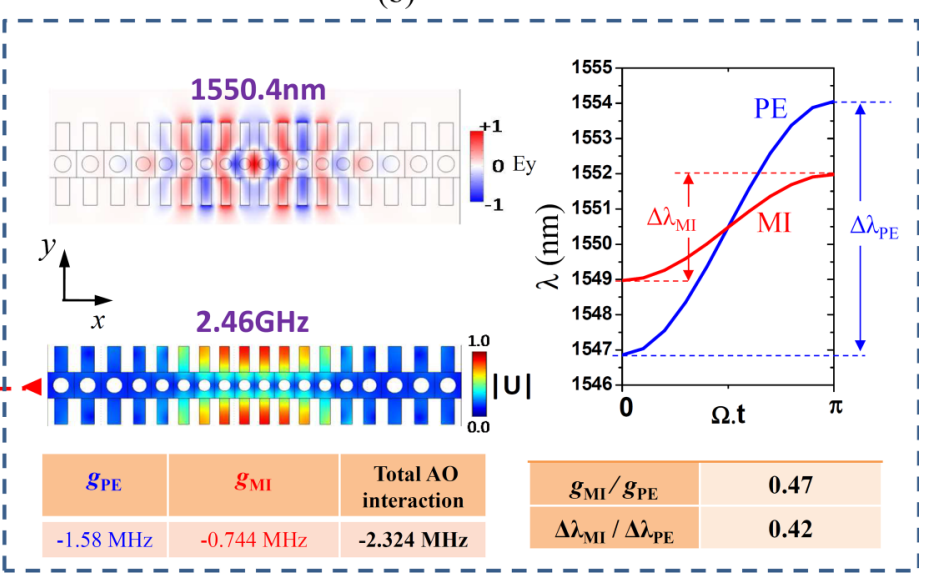

FIG. 4. (Color online) (a) Phononic band structure of the perfect nanobeam where the red shaded regions named BG1, BG2, and BG3 are the gaps for EE symmetry modes. (b) AO coupling between the photonic cavity mode at $1550.4 \mathrm{~nm}$ and the phononic mode at $2.46 \mathrm{GHz}$, estimated by the wavelength shift modulation along half of the acoustic period, and by the calculation of the coupling coefficients $g_{\mathrm{PE}}$ and $g_{\mathrm{MI}}$.

the plane $y-z$, which is perpendicular to the nanobeam at the cavity center. Again, a nonvanishing AO coupling requires that the phononic mode have an even symmetry with respect to this plane, so we can refer to it as an EEE mode. Our study focuses then only on the EE phononic cavity modes having this specific supplementary symmetry criterion in the range of 1 to $6.5 \mathrm{GHz}$. Figure 4(a) reminds the band structure of the perfect nanobeam in which we highlighted in red the phononic modes having EE symmetry. Three gaps for this symmetry are found which are indicated by red shaded regions between 1 and $6.5 \mathrm{GHz}$. Let us notice that only the gap BG2 overlaps with the absolute band gap. In the following, we focus only on the case of cavity with tapering $\alpha$ as the same conclusions hold for the tapering $\beta$.

We searched for all existing confined EEE phononic modes within the cavity and found several of them that can couple with the photonic mode with a total optomechanical coupling coefficient less than $0.6 \mathrm{MHz}$ apart from one phonon which gives the highest coupling evaluated at $2.324 \mathrm{MHz}$ with 0.744 and $1.58 \mathrm{MHz}$ contributions of the MI and PE effects respectively [Fig. 4(b)]. This coupling rate value is in the order of high coupling rates reported in previous works $(2 \pi \times 292 \mathrm{kHz}$ [18]; $2 \pi \times 320 \mathrm{kHz}$ [26] and $2 \pi \times 860 \mathrm{kHz}$ [23]). The sign of the coupling coefficient is very important to know whether the photoelastic and the interface motion mechanisms contribute in phase or out of phase to the total optomechanic coupling for each phonon-photon pair. However, it does not have any meaning when comparing between two phonon-photon pairs.

This confined phonon found is located at $2.46 \mathrm{GHz}$ which is not inside any of the three gaps related to EE symmetry modes highlighted in Fig. 4(a); this means that we have here a resonant mode rather than a localized cavity mode and, in turn, its quality factor is relatively poor, equal to $1.3 \times$ $10^{3}$, which is in the same order of what Eichenfield et al. (see related supplementary information in [21]) found in their structure. So, below we shall change the tapering of the cavity to push this mode inside a phononic gap. Especially, it is worthwhile noticing that the high AO coupling rate of this mode is probably related to the shape of the displacement field, namely a breathing motion of the stubs located around the center of the cavity which move all in phase.

In addition to the calculation of the coupling coefficients, we also show in Fig. 4(b) the modulation of the photonic cavity mode wavelength by the phononic mode at several selected instants along half of the acoustic period $(0<\Omega t<$ $\pi$ where $\Omega$ is the acoustic frequency) as the other half (i.e., $\pi<\Omega t<2 \pi$ ) can be deduced by symmetry. This equivalent method described in the beginning of this section is applied to both MI and PE effects and gives a clear description of how the photonic wavelength is shifted by the acoustic strain. We also point out that, for the sake of the numerical simulations, we have fixed the maximum value of the strain to $1 \%$. As we have explained in [25], this $1 \%$ value of the strain is relatively large as compared to more realistic values of $0.01 \%$ to $0.001 \%$ that can be expected from an acoustic source exciting the phononic cavity mode, but the photon frequency modulation is proportional to this value as far as the phonon-photon coupling is a first-order process. From Fig. 4(b), the calculation method shows that we are in the presence of a sinusoidal modulation of the photonic wavelength along the phononic vibration period with 7.2- and 3-nm amplitudes for PE and MI effects respectively. This makes a total of 10.2-nm amplitude in the wavelength shift for $1 \%$ maximum strain. Then, we can deduce that this shift will be in the order of $0.1 \mathrm{~nm}(0.01 \mathrm{~nm})$ for a $0.01 \%(0.001 \%)$ strain. Furthermore, as we compare the $g_{\mathrm{MI}} / g_{\mathrm{PE}}$ with the ratio $\Delta \lambda_{\mathrm{MI}} / \Delta \lambda_{\mathrm{PE}}$ between the amplitudes of the optical wavelength modulation of the two effects, we can find then good agreement between the coupling coefficients calculation and the time acoustic modulation of the photonic wavelength. Let us notice that the modulation amplitude is given here in units of $\mathrm{nm}$ which is suitable to compare the shift of the photonic mode with its width and location which are usually expressed in $\mathrm{nm}$. In contrast, the coupling coefficient derived in the previous section was expressed in $\mathrm{MHz}$ which is suitable for comparison with the values usually reported in the literature. Obviously, one can relate the wavelength unit with the frequency unit by using the relation $\omega=2 \pi c / \lambda$. Nevertheless, as concerns the orders of magnitude reported above, it is worth mentioning that in the literature, the coupling 


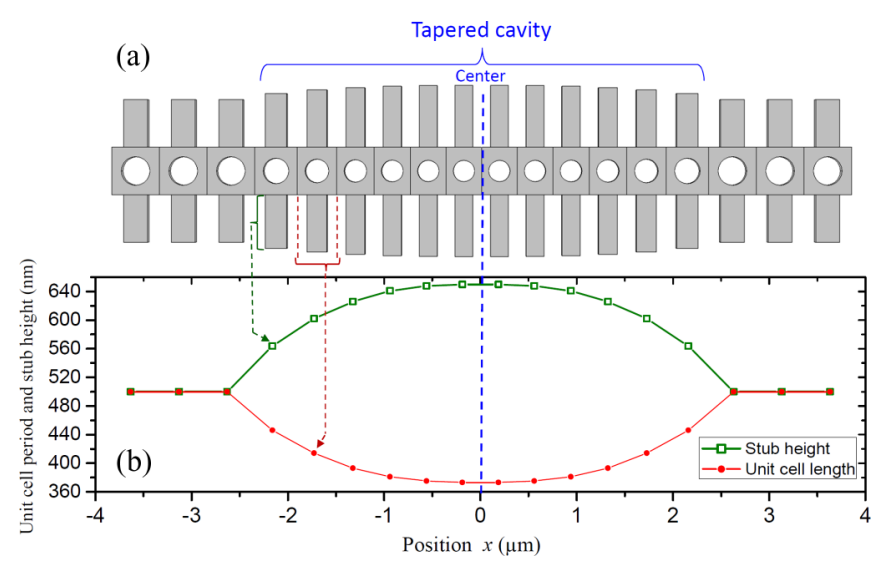

FIG. 5. (Color online) (a) Schematic view of the cavity created inside the phoxonic strip by tapering the unit-cell period, the hole's radius, as well as the lateral stub's height. (b) The unit-cell period and lateral stub heights profiles from cell to cell indicated in red circles and green squares, respectively, outside and inside the cavity.

coefficient generally defines the shift of the photonic mode imparted to the zero-point motion of an effective oscillator, whereas in the calculation of the modulation, an arbitrary value of the acoustic strain is assumed.

One can define a figure of merit by dividing the total OM coupling coefficient by half of the full width at half maximum (FWHM) of the photonic mode peak, so as to have a quantitative estimation of the photonic peak shift compared to its width. The absolute value of this quantity is not meaningful due to the arbitrariness of the acoustic strain chosen for the calculation, but it may be useful for the sake of comparison between different structures. We will denote this ratio as FOM in the paper. For the case of photon-phonon pair of Fig. 4 for instance, we obtain a ratio of $4.67 \times 10^{-3}$.

As mentioned above, to increase the confinement of the resonant phononic mode at $2.46 \mathrm{GHz}$ as well as its quality factor, it is necessary to shift it inside one of the EE symmetry gaps. The most suitable would be to shift it downwards inside the nearest gap called BG1 which is a gap of EE symmetry although it is not a complete gap. Below, we also briefly discuss the possibility of shifting this phononic mode towards the complete band gap BG2 situated at a much higher frequency (around $4 \mathrm{GHz}$ ). We considered then a new tapered cavity by changing gradually not only the periods' and holes' radii from the cavity center as in Fig. 2, but also by increasing this time the lateral stubs' heights from the side of the cavity towards the middle as presented in Fig. 5, knowing that the acoustic mode dispersion depends strongly on the stub height.

For the new tapered cavity presented in Fig. 5, we have performed the same photonic investigation as in the previous structure presented in Fig. 2. The photonic mode we are dealing with in this case keeps the same field distribution as the one studied before. However, the stub tapering makes its wavelength to be located at $1605.2 \mathrm{~nm}$ instead of $1550.4 \mathrm{~nm}$ found in the first structure with tapering $\alpha$. The QF of this mode is estimated at $1.75 \times 10^{5}$ in this case. Regarding the acoustic dispersion behavior of this new cavity, we also searched for the new localized phononic modes that can have strong coupling with the photonic cavity mode. Figure 6 summarizes the obtained results as we point out the main phononic cavity modes found that couple strongly with the photon and their total corresponding $\mathrm{AO}$ coupling coefficients.

In the case of this new structure, we found three phononic cavity modes with EE symmetry giving the highest coupling coefficient with the studied photonic mode. These modes are presented in Fig. 6(a) and are localized inside the gaps corresponding to their EE symmetry. In fact, the wave field of the lowest phononic mode located at $2.08 \mathrm{GHz}$ which is inside the band gap BG1 looks like the localized mode found in the case of the previous structure at $2.46 \mathrm{GHz}$ less confined and outside the gap (Fig. 4). So, as we tapered the stubs of the cavity, we were able to shift down this particular phononic mode to lower its frequency (i.e., from 2.46 to $2.08 \mathrm{GHz}$ ) inside the gap BG1 and make it more efficiently confined. Acoustic transmission calculation showed that its acoustic QF is increased to more than $3 \times 10^{6}$. Furthermore, two other confined EE acoustic modes were found at higher frequencies inside the gap called BG3 at 5.34 and $5.49 \mathrm{GHz}$ with high AO coupling coefficients of about 1.2 and $1.064 \mathrm{MHz}$ respectively [see Fig. 6(b)]. The QF of these two defect phononic modes is higher than $10^{6}$. We can then conclude that localizing phononic modes inside the gap promote having better confinement for high acoustic QF with strong AO coupling. (a)

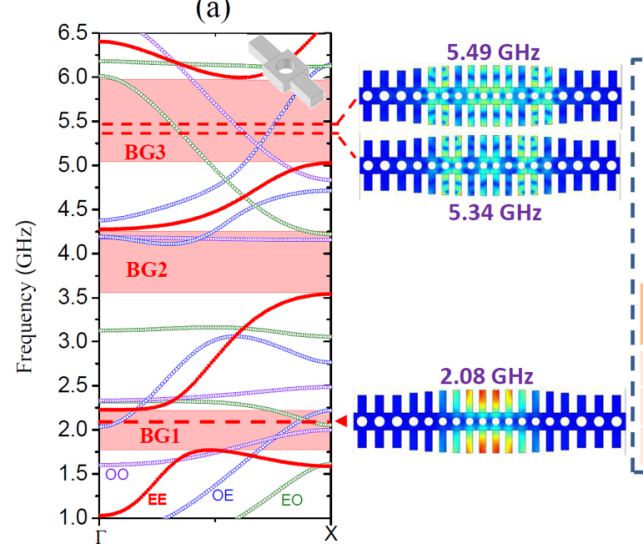

(b)

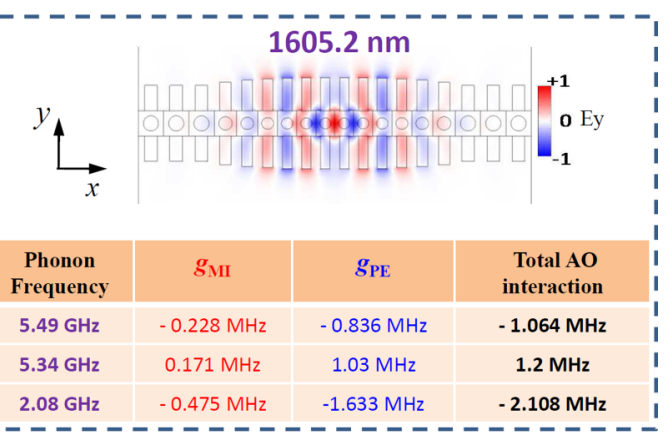

FIG. 6. (Color online) (a) Phononic band structure of the perfect nanobeam. (b) AO coupling between the photonic cavity mode at $1605.2 \mathrm{~nm}$ and the phononic modes at $2.08,5.34$, and $5.49 \mathrm{GHz}$, estimated by the calculation of the coupling coefficients $g_{\mathrm{PE}}$ and $g_{\mathrm{MI}}$. 


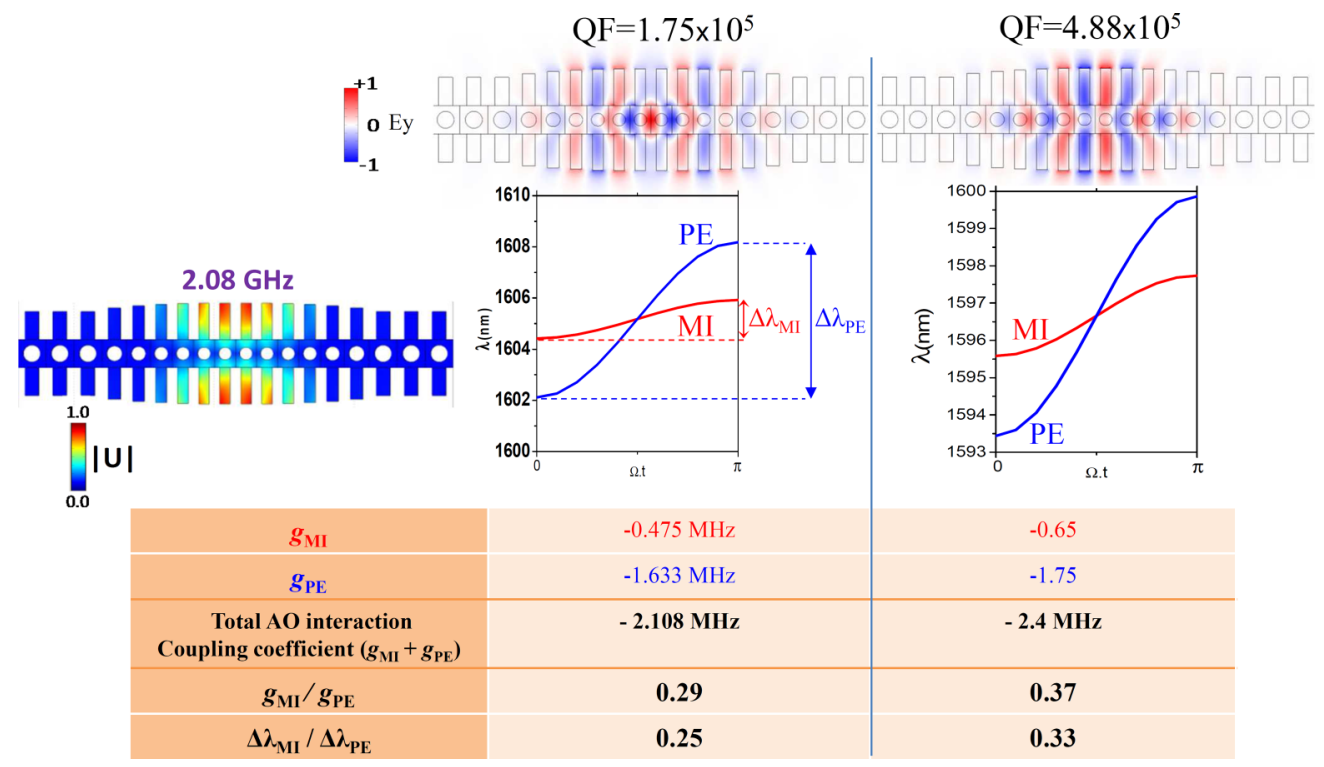

FIG. 7. (Color online) AO coupling between the photonic cavity mode at $1605.2 \mathrm{~nm}$ as well as the one at $1596.7 \mathrm{~nm}$ and the phononic modes at $2.08 \mathrm{GHz}$, estimated by the calculation of the coupling coefficients $g_{\mathrm{PE}}$ and $g_{\mathrm{MI}}$ as well as their wavelength modulation by the acoustic field.

We remind of the fact that the phoxonic cavity studied here gives six well localized OE photonic cavity modes inside the gap with high QF. We found out that the same phononic modes indicated in Fig. 6 can couple strongly with all these photonic modes as the calculation of the coupling coefficients showed. The example presented in Fig. 7 gives the results of the modulation of two OE photonic modes' wavelength by the phononic cavity mode at $2.08 \mathrm{GHz}$ inside the gap BG1 indicated in Fig. 6 for a $1 \%$ maximum strain. We have also given the coupling coefficients' values in the same figure for the sake of comparison between the two methods used for the estimation of the $\mathrm{AO}$ coupling strength.

As we can see in Fig. 7, the two photons presented here couple strongly with the phononic cavity mode at $2.08 \mathrm{GHz}$ with coupling coefficient higher than $2 \mathrm{MHz}$ which is in the same order of high coupling rates reported in previous works $[18,26,23]$. The calculations show that we are in the presence of a sinusoidal modulation of the photonic wavelength by the two MI and PE effects which contribute additively in the total AO interaction. Furthermore, one can notice that the $\mathrm{PE}$ effect is the strongest with at least $70 \%$ contribution in the total AO coupling effect compared to the MI effect for the photon at $1605.2 \mathrm{~nm}$ and $60 \%$ in the case of photon at $1596.7 \mathrm{~nm}$. Furthermore, as we compare the $g_{\mathrm{MI}} / g_{\mathrm{PE}}$ with the ratio $\Delta \lambda_{\mathrm{MI}} / \Delta \lambda_{\mathrm{PE}}$ between the amplitudes of the optical wavelength modulation of the two effects, good agreement can then be found between the coupling coefficient calculations and the time acoustic modulation of the photonic wavelength and their additive contribution in the total $\mathrm{AO}$ interaction.

For the sake of optomechanic comparison between the two photonic modes, we calculate the ratio FOM which is found to be of $3.95 \times 10^{-3}$ for the mode at $1605.2 \mathrm{~nm}$ while it is of $12.48 \times 10^{-3}$ for the photon at $1596.7 \mathrm{~nm}$. One can then see from Fig. 7 that even if the total coupling coefficient is a little higher in the case of photon $1605.2 \mathrm{~nm}$ than the one of photon $1596.7 \mathrm{~nm}$, the latter has higher FOM (three times higher).
And this is understandable as the QF of this mode is $4.88 \times$ $10^{5}$ which is more than twice higher that the $\mathrm{QF}$ of the mode at $1596.7 \mathrm{~nm}\left(1.75 \times 10^{5}\right)$. Then, one can make the comparison of the optomechanic strength between the photon-phonon pairs by evaluating the defined figure of merit FOM quantity. The latter becomes small if the photonic mode has low QF and/or low optomechanic coupling.

In the above discussion, the phononic mode with a high AO coupling rate was pushed downwards inside the BG1 gap which is a partial gap with EE symmetry. However, in practice, such a phononic mode may present a leakage out of the cavity due to unwanted imperfections resulting from the microfabrication processes that cannot be avoided and produce a departure from a perfect symmetrical structure. For this reason, having a localized phononic mode inside a

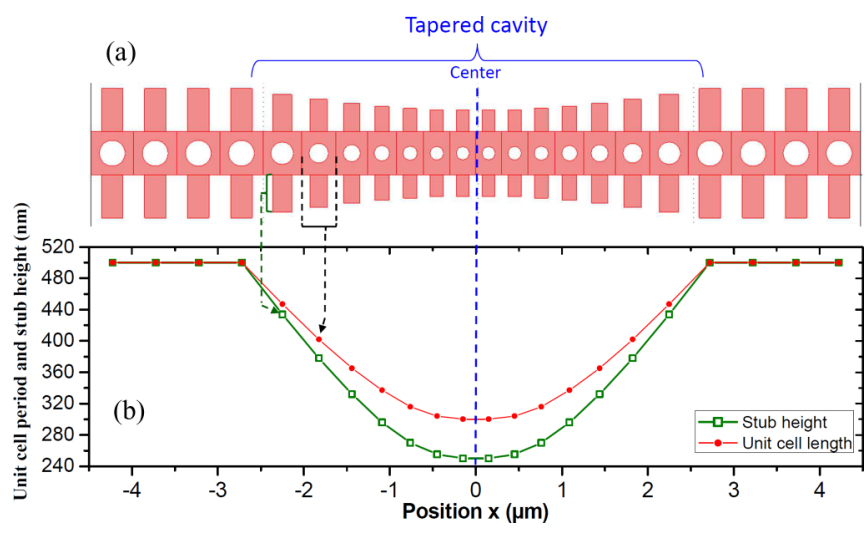

FIG. 8. (Color online) (a) Schematic view of the cavity created inside the phoxonic strip by tapering the unit-cell period, the hole's radius, as well as reducing the lateral stub's height. (b) The unit-cell period and lateral stub heights profiles from cell to cell are indicated in red circles and green squares, respectively, outside and inside the cavity. 
(a)

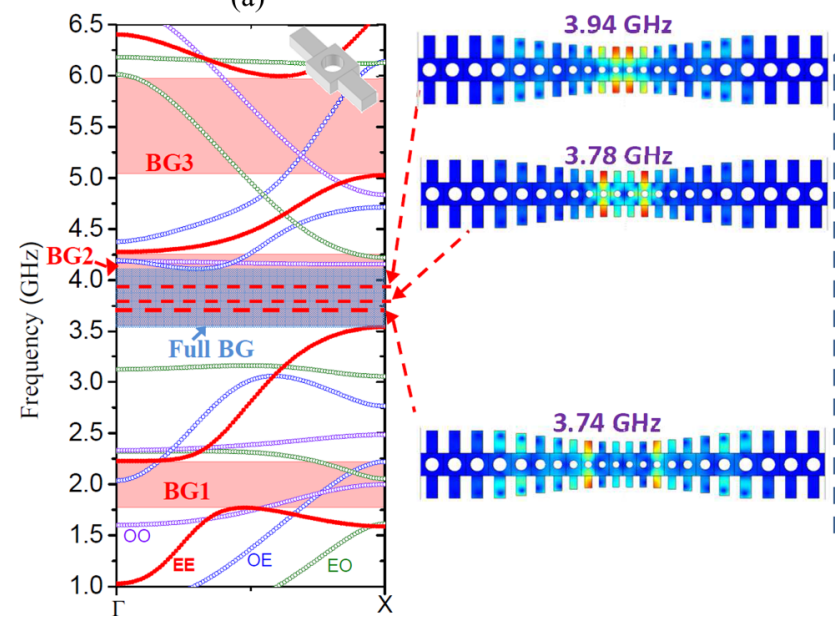

(b)

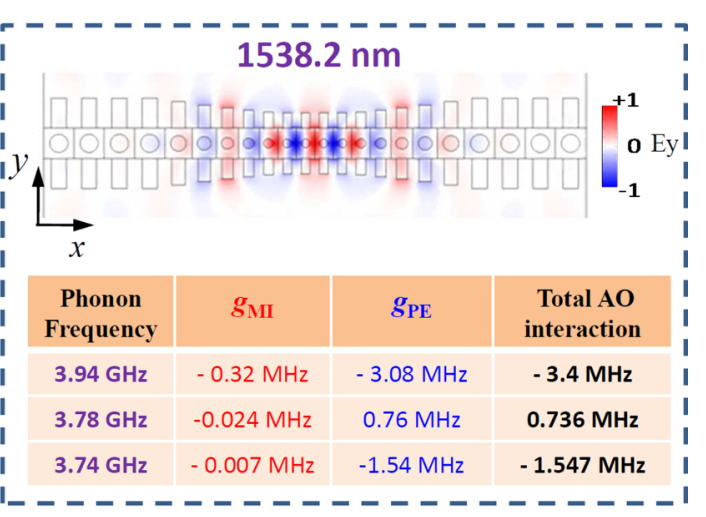

FIG. 9. (Color online) (a) Phononic band structure of the perfect nanobeam. (b) AO coupling between the photonic cavity mode at $1538.2 \mathrm{~nm}$ and the phononic modes at $3.74,3.78$, and $3.94 \mathrm{GHz}$, estimated by the calculation of the coupling coefficients $g_{\mathrm{PE}}$ and $g_{\mathrm{MI}}$.

complete gap would be desirable (see Gomis-Bresco et al. [34]). In the following, we show an example of the tapered cavity where the mode at $2.46 \mathrm{GHz}$ has been pushed upwards inside the complete BG2 gap. The drawback is that because this gap is relatively far from the original phononic mode, the tapering of the cavity should be realized with a faster variation of the parameters and therefore the quality factors of the corresponding photonic modes become much lower than in the previous example.

The tapered cavity considered here consists of decreasing gradually the heights of the stubs at the same time that we change the lattice parameter and holes radii (Fig. 8).

In this structure, a photonic mode with the same field characteristics as those presented in Figs. 3(a) and 6(b) appears now at $1538.2 \mathrm{~nm}$ with a quality factor estimated around $3 \times$ $10^{3}$. Figure 9 gives the phononic modes appearing inside the complete gap, together with the maps of their displacement field and their AO coupling rates with the photonic mode. The phononic mode corresponding to the breathing motion of the stubs at the cavity center happens now at $3.94 \mathrm{GHz}$ and gives rise to a high AO coupling coefficient of $3.4 \mathrm{MHz}$. Two other localized modes exist also in the band gap with lower AO coupling. For all these phononic modes, the QF is higher than $10^{6}$ and the main contribution to the AO coupling comes from the photoelastic mechanism whereas the MI effect remains relatively small.

Finally, let us point out that the phoxonic cavity studied in this paper has its center between two holes, but it could be also possible to consider the other case where the cavity is constructed such that its center coincides with the center of the hole while adopting the same tapering. In fact, we have performed similar calculations for this geometry by considering the same tapering configuration over the unit cells of the cavity. We showed that we can find new OE optical modes with high QF (over $10^{5}$ ) inside the photonic gap. In addition, the same symmetry analysis demonstrates that only phononic cavity modes with EEE symmetry can couple strongly with the photonic modes. Equivalent results were obtained in this case in which we found a similar EEE phononic mode giving strong AO coupling, shifted from $2.5 \mathrm{GHz}$ outside the gap (no tapering over the stubs height) into $2.079 \mathrm{GHz}$ inside the BG1 gap by tapering the cavity over the stubs' height. For example, this acoustic cavity mode gives total AO coupling coefficients of 2.46 and $2.23 \mathrm{MHz}$ respectively for two photonic cavity modes located at 1601.6 and $1611.7 \mathrm{~nm}$.

\section{CONCLUSIONS}

In this paper, we have investigated the AO interaction in a corrugated phoxonic silicon nanobeam presenting band gaps both for photonic and phononic modes. By creating a well-designed tapered cavity, we were able to create highly confined phononic and photonic modes with high QF inside the gap which can then enhance their AO interaction. We have shown that this tapering can be adjusted to shift either the photon wavelength or the phonon frequency of interest in the suitable targeted wavelength or frequency range which can be useful for experimental AO phenomena observation and characterization. In fact, by changing the tapering rates over holes and the stubs, we have shown that optical modes' wavelength can be shifted, and acoustic modes' frequency can be tuned to place them inside the desired gap by changing the height of the stubs. This allows more confinement to reach a high mechanical quality factor (more than $10^{6}$ ). Regarding the AO coupling, particular attention was drawn to the symmetry of the acoustic modes to focus only on the ones that can couple strongly with the trapped photonic modes, namely phononic modes having even symmetry wave fields with respect to the geometry's symmetries. The AO coupling was studied in the case of two photonic modes and its strength was estimated by means of two methods: the calculation of the coupling coefficients in one hand, and the acoustic modulation of photonic wavelength that gives a sinusoidal shape along the acoustic period on the other hand. Good agreement was found by these two methods. High coupling rates were found of $2 \mathrm{MHz}$ with photonic cavity modes displaying QF of $10^{5}$ and even values up to $3.4 \mathrm{MHz}$ but with smaller photonic quality factors. The phoxonic strip waveguide offers many possibilities to tune the photonic wavelengths and phononic frequency modes inside the complete band gap to increase the 
confinement efficiency and overcome wave leaky problems caused by the microfabrication imperfection. It can then serve as an efficient optomechanical tool for electromagnetic waves control by phonons.

\section{ACKNOWLEDGMENT}

The authors acknowledge the support of the European Commission Seventh Framework Programs (FP7) under the FET-Open project TAILPHOX N 233883.
[1] E. Yablonovitch, J. Opt. Soc. Am. B 10, 283 (1993).

[2] T. F. Krauss, R. M. De la Rue, and S. Brandt, Nature (London) 383, 699 (1996).

[3] M. M. Sigalas and E. N. Economou, J. Sound Vib. 158, 377 (1992).

[4] M. S. Kushwaha, P. Halevi, L. Dobrzynski, and B. DjafariRouhani, Phys. Rev. Lett. 71, 2022 (1993).

[5] Y. Pennec, J. O. Vasseur, B. Djafari-Rouhani, L. Dobrzynski, and P. A. Deymier, Surf. Sci. Rep. 65, 229 (2010).

[6] M. Maldovan and E. L. Thomas, Appl. Phys. B 83, 595 (2006).

[7] S. Sadat-Saleh, S. Benchabane, F. I. Baida, M. P. Bernal, and V. Laude, J. Appl. Phys. 106, 074912 (2009).

[8] S. Mohammadi, A. A. Eftekhar, A. Khelif, and A. Adibi, Opt. Express 18, 9164 (2010).

[9] Y. Pennec, B. Djafari-Rouhani, E. H. El Boudouti, C. Li, Y. El Hassouani, J. O. Vasseur, N. Papanikolaou, S. Benchabane, V. Laude, and A. Martinez, Opt. Express 18, 14301 (2010).

[10] Y. El Hassouani, C. Li, Y. Pennec, E. H. El Boudouti, H. Larabi, A. Akjouj, O. Bou Matar, V. Laude, N. Papanikolaou, A. Martinez, and B. Djafari Rouhani, Phys. Rev. B 82, 155405 (2010).

[11] Y. Pennec, B. Djafari-Rouhani, E. H. El Boudouti, C. Li, Y. El Hassouani, J. O. Vasseur, N. Papanikolaou, S. Benchabane, V. Laude, and A. Martinez, Chin. J. Phys. 49, 100 (2011).

[12] D. Bria, M. B. Assouar, M. Oudich, Y. Pennec, J. Vasseur, and B. Djafari-Rouhani, J. Appl. Phys. 109, 014507 (2011).

[13] A. H. Safavi-Naeini, J. T. Hill, S. Meenehan, J. Chan, S. Groeblacher, and O. Painter, Phys. Rev. Lett. 112, 153603 (2014).

[14] V. Laude, J. C. Beugnot, S. Benchabane, Y. Pennec, B. Djafari-Rouhani, N. Papanikolaou, J. M. Escalante, and A. Martinez, Opt. Express 19, 9690 (2011).

[15] M. Maldovan and E. L. Thomas, Appl. Phys. Lett. 88, 251907 (2006).

[16] I. E. Psarobas, N. Papanikolaou, N. Stefanou, B. DjafariRouhani, B. Bonello, and V. Laude, Phys. Rev. B 82, 174303 (2010).

[17] Q. Rolland, M. Oudich, S. El-Jallal, S. Dupont, Y. Pennec, J. Gazalet, J. C. Kastelik, G. Lévêque, and B. Djafari-Rouhani, Appl. Phys. Lett. 101, 061109 (2012).

[18] A. H. Safavi-Naeini and O. Painter, Opt. Express 18, 14926 (2010).
[19] D. A. Fuhrmann, S. M. Thon, H. Kim, D. Bouwmeester, P. M. Petroff, A. Wixforth, and H. J. Krenner, Nat. Photon. 5, 605 (2011).

[20] E. Gavartin, R. Braive, I. Sagnes, O. Arcizet, A. Beveratos, T. J. Kippenberg, and I. Robert-Philip, Phys. Rev. Lett. 106, 203902 (2011).

[21] M. Eichenfield, J. Chan, R. M. Camacho, K. J. Vahala, and O. Painter, Nature (London) 462, 78 (2009).

[22] M. Eichenfield, J. Chan, A. H. Safavi-Naeini, K. J. Vahala, and O. Painter, Opt. Express 17, 20078 (2009).

[23] J. Chan, A. H. Safavi-Naeini, J. T. Hill, S. Meenehan, and O. Painter, Appl. Phys. Lett. 101, 081115 (2012).

[24] S. El-Jallal, M. Oudich, Y. Pennec, B. Djafari-Rouhani, V. Laude, J.-C. Beugnot, A. Martínez, J. M. Escalante, and A. Makhoute, Phys. Rev. B 88, 205410 (2013).

[25] S. El-jallal, M. Oudich, Y. Pennec, B. Djafari-Rouhani, A. Makhoute, Q. Rolland, S. Dupont, and J. Gazalet, J. Phys.: Condens. Matter 26, 015005 (2014).

[26] A. H. Safavi-Naeini, T. P. Mayer Alegre, M. Winger, and O. Painter, Appl. Phys. Lett. 97, 181106 (2010).

[27] K. Srinivasan and O. Painter, Opt. Express 11, 579 (2003).

[28] Y. Akahane, T. Asano, B.-S. Song, and S. Noda, Nat. London 425, 944 (2003).

[29] B.-S. Song, S. Noda, T. Asano, and Y. Akahane, Nat. Mater. 4, 207 (2005).

[30] E. Kuramochi, M. Notomi, S. Mitsugi, A. Shinya, T. Tanabe, and T. Watanabe, Appl. Phys. Lett. 88, 041112 (2006).

[31] E. Kuramochi, H. Taniyama, T. Tanabe, A. Shinya, and M. Notomi, Appl. Phys. Lett. 93, 111112 (2008).

[32] Y. Tanaka, T. Asano, and S. Noda, J. Lightwave Technol. 26, 1532 (2008).

[33] Y. Pennec, B. Djafari-Rouhani, C. Li, J. M. Escalante, A. Martinez, S. Benchabane, V. Laude, and N. Papanikolaou, AIP Adv. 1, 041901 (2011).

[34] J. Gomis-Bresco, D. Navarro-Urrios, M. Oudich, S. El-Jallal, A. Griol, D. Puerto, E. Chavez, Y. Pennec, B. Djafari-Rouhani, F. Alzina, A. Martínez, C. M. Sotomayor Torres, arXiv:1401.1691 [Nat. Commun. (to be published)].

[35] P. B. Deotare, M. W. McCutcheon, I. W. Frank, M. Khan, and Marko Lončar, Appl. Phys. Lett. 94, 121106 (2009).

[36] M. Oudich, S. El-Jallal, Y. Pennec, G. Lévêque, B. DjafariRouhani, Q. Rolland, S. Dupont, J. Gazalet, J. C. Kastelik, and A. Makhoute, International Ultrasonics Symposium (IUS), Dresden, Germany (IEEE, Piscataway, NJ, 2012), pp. 2466-2469. 